\title{
Come ricorderemo il 2020? Et voilà: PUF!
}

\author{
Marco Lombardi \\ Editor in Chief, Giornale di Clinica Nefrologica e Dialisi, Firenze - Italy
}

"Visto, papà? Si comincia bene. L'uomo del futuro è una donna." (1)

Come un anno orribile!

Ma è proprio tutta colpa di questo benedetto anno bisestile?

Non credo proprio, cerchiamo di essere obiettivi, sinceri, ragionevoli.

L'anno è funestato sin da poco dopo il suo inizio (nel nostro Paese) dalla diffusione di questo SARS virus (severe acute respiratory syndrome), che, a onor del vero, non è certo il primo della famiglia ad affacciarsi nell'essere umano.

Come poi si è sviluppata questa infezione sino ai giorni attuali di marzo 2021 lo sappiamo tutti. Quello che forse non è chiaro a tutti è come sono andate le cose prima, cosa veramente conta e cosa no, ripeto siamo obiettivi, sinceri e ragionevoli.

Per esempio, non tutti i Paesi hanno amministrato la diffusione della trasmissione dell'infezione allo stesso modo: Occidente vs Oriente!

Se, a Oriente, già verso la fine del 2019 si era capito, non è facilmente comprensibile, specialmente con il senno del poi, perché l'Occidente abbia sottovalutato così tanto e così a lungo questo pericolo.

Ma questo è il passato prossimo. Guardiamo un po' indietro e, soprattutto, proviamo a guardare dove si doveva, si deve e si dovrà guardare: alla situazione ambientale del nostro Pianeta!

Può bastare al 2015? Quando la scienza aveva piena contezza della presenza di svariati Corona virus presenti in animali che vivevano nei propri spazi vitali, che se non fossimo andati a ridurre/invadere non avrebbero concesso il cosiddetto "spillover"?

Received: March 18, 2021

Accepted: March 19, 2021

Published online: April 12, 2021

Indirizzo per la corrispondenza:

Marco Lombardi

Ospedale del Mugello

Via Della Resistenza, 60

50032 Borgo San Lorenzo, Firenze - Italy

lombardim@tin.it
No. Nel 2012 un altro Corona virus aveva già causato un'epidemia (MERS), fortunatamente contenuta grazie proprio alla sua gravità ... Nel 2010 un virus influenzale aveva già fatto una cosa simile ... Nel 2002-3 un altro Corona (SARS-CoV-1) era già passato all'umano provocando circa un migliaio di decessi tra i sanitari ... E, tra il 2002 e il 1997, si erano verificate varie epidemie localizzate di aviaria ... Prima? ... Prima si può arrivare al 1957 con l'Asiatica ... 63 anni fa!

I prodromi c'erano, bastava non ignorarli.

Cercherò, nel resto di questo articolo, di mettere in correlazione l'infezione (che ha determinato il ricordo del 2020 in tutti noi) con l'ambiente (il vero determinante di questi ricordi).

Come ha argomentato il Professor Ernesto Burgio in diversi webinar tuttora presenti in rete (2) e che ringrazio per avermi aperto almeno un po' la mente, l'infezione da Covid nelle forme gravi ha sempre come substrato dei meccanismi immunopatogenetici, vale a dire delle reazioni immunomediate "eccessive", al pari di una malattia autoimmune, a spiegare che la sindrome immunoinfiammatoria del Covid-2 con la sua tempesta citochimica produce endotelite e tromboembolismi sistemici e coagulazione intravascolare disseminata (3).

Teniamo a mente questo termine: endotelite (infiammazione delle cellule endoteliali, vale a dire quelle che rivestono la superfice interna di tutti i nostri vasi circolatori).

Cercherò, da qui in poi, di argomentare l'azione sinergica tra trigger infettivo (Covid-2, per esempio) e trigger ambientale e la loro stretta correlazione.

Risale al 2014 l'osservazione della correlazione tra incidenza di una malattia autoimmune (vale a dire con produzione endogena di anticorpi contro una parte di noi) scatenata da agenti infettivi e i venti (sì, l'aria che si muove, non il numero!!) provenienti da specifiche aree geografiche $(4,5)$. Quindi, di malattie in grado di provocare un'endotelite acuta, vale a dire una vasculite immunoinfiammatoria, in grado di danneggiare gravemente le pareti arteriolari del nostro corpo.

Entra in scena finalmente (purtroppo) l'attore principale: il particolato fine e ultra fine (PUF), che dell'inquinamento atmosferico è il Re!

II PUF è, infatti, ormai lo sappiamo, ma sembriamo volerlo ignorare, uno dei principali determinanti dello stato 
infiammatorio sistemico che ci accompagna per gran parte della vita e che apre la strada ad aterosclerosi e, quindi, a malattie cardiovascolari, ma anche autoimmuni (siano queste immunomediate o allergiche), endocrino-metaboliche (obesità e diabete mellito-2), neurodegenerative (sclerosi multipla, SLA o sclerosi laterale amiotrofica, Parkinson, Alzheimer, autismi) e, per finire, tumorali. Tutte patologie in grande aumento e sicuri fattori di comorbilità all'origine della quasi totalità dei casi gravi di Covid-2.

II PUF, per le sue dimensioni submicroscopiche, è in grado di superare tutte le barriere umane, anatomiche e funzionali (6-8): le membrane cellulari (anche degli alveoli polmonari, per entrare nel torrente circolatorio), le membrane cellulari anche delle nostre linee cellulari deputate all'organizzazione delle nostre difese (p. es., macrofagi ...), le membrane nucleari delle nostre cellule, dove risiede il nostro genoma, la nota barriera emato-encefalica (l'ingresso al nostro sistema nervoso centrale o SNC) e, persino, la barriera placentare ... Sì, oggi è considerato l'inquinante più pericoloso anche e proprio per le sue capacità di indurre variazioni EPIGENETICHE ... $\mathrm{E}$, infine, entra anche nelle cellule gametiche, quelle deputate alla riproduzione e al passaggio di informazioni genetiche per la nostra progenie!

Il PUF può rimanere in sospensione nell'aria per diversi giorni ed essere trasportato per migliaia di chilometri e, quindi, non è presente solo nelle nostre grandi città. Inoltre il PUF è in grado di adsorbire (legare) sulla sua superfice altre sostanze come diossine, metalli pesanti, spore, batteri e virus (6), ma, attenzione, è dimostrato che i virus sono spesso parti e sono perlomeno inattivati ... Pertanto, non è come si era paventato in principio che la maggiore morbilità nelle zone maggiormente inquinate come la Padania e alcune del nord della Lombardia fosse dovuto al trasporto del virus nei polmoni per l'alta concentrazione di PUF che lo lega, ma, come vedremo dopo, è perché il PUF è un fattore pro-infiammatorio, ecco l'arcano.

Sebbene il PUF entri nell'organismo attraverso i polmoni, poi va a colpire più duramente gli endoteli e le cellule della circolazione dei vari organi, come cuore, reni, fegato e SNC, accumulandosi nelle loro cellule endoteliali e provocando uno stato infiammatorio cronico, latente e subclinico, di bassa intensità ma continuo, che apre la strada all'aterosclerosi e alle alterazioni pro-trombotiche.

L'esposizione protratta al PUF (come, per esempio, nelle persone meno giovani, vale a dire in quelle che hanno respirato più a lungo PUF) è verosimilmente il fattore trigger non solo a tutto quanto già scritto ma anche alla sindrome infettiva che produce Covid-2. Questo virus (come altri, del resto) apre la strada a una riacutizzazione di un'endotelite cronica già presente e subclinica da inquinamento, che, così, diviene rapidamente iperacuta e sistemica, vale a dire quella tipica delle forme più gravi di Covid-2 $(9,10)$.

È chiaro, quindi, che non è tanto l'età dei nostri cari che il Covid-2 si è portato via il maggior fattore di rischio per il
Covid, ma è, viceversa, l'infiammazione cronica endoteliale delle loro arterie. E questa è data dal carico di inquinanti ambientali a cui fino a meno di 100 anni fa l'essere umano non era esposto e purtroppo abituato. Paradossalmente, infatti, nei grandi anziani di oltre 90 anni che sono vissuti e vivono in aree a minor carica di PUF, il rischio di gravi forme di Covid-2 è minore (2). Ed ecco che ciò ci viene in aiuto per spiegare la maggiore mortalità da Covid-2 nelle zone del nord Italia, dove l'inquinamento ambientale è notoriamente più elevato, anzi il più elevato d’Europa $(2,10)$.

Eccoci al dunque, non tanto dell'anno 2020 ma dei precedenti e, purtroppo, dei seguenti, se non cambierà qualcosa: le condizioni critiche della biosfera e degli ecosistemi.

La vera cura del Covid-2 e delle prossime previste epidemie risiede nel ridurre in tempi rapidissimi l'enorme inquinamento prodotto dall'uomo, con i suoi impianti industriali ed ogni tipo di traffico veicolare, vale a dire la produzione giornaliera di svariate tonnellate di PUF. La malattia del Pianeta è questa, e i cambiamenti climatici, le crisi ecosistemiche e le pandemie non sono altro che i sintomi, diciamo clinici, di questa malattia. Farmaci, vaccini e distanziamenti sono equiparabili a un mero trattamento sintomatico (sono poco più che un'aspirina o un antidolorifico ...). Certo, per amor di Dio, trattamenti, necessari e utilissimi, ma la chiave del problema è altrove, ce lo dice il Pianeta ormai da oltre 50 anni. Dobbiamo aspettare la prossima pandemia, il prossimo cataclisma, la prossima estinzione tra le tante annunciate, per capirlo?

Per concludere, il 2020, quest'anno orribile, non lo ricorderemo solo per la pandemia Covid-2 (11). Dovremo ricordarcelo anche come l'anno in cui sono state trovate per la prima volta nella placenta di esseri umani sani tracce di microplastiche, come a dire che nascono i primi bambini-cyborg, cioè composti non solamente da cellule umane, ma da una "mistura" di componente biologica (la nostra) e di componente antropocenica (inorganica di plastiche, come il propilene, vale a dire il materiale delle nostre bottiglie e dei tappi di plastica, per esempio) (12).

Ma non è tutto qui, il 2020 dovrà essere ricordato anche per essere l'anno in cui la cosiddetta massa antropica (quella creata dall'uomo, o human made-mass) ha superato la biomassa vivente (quella del Pianeta, vale a dire quella animale e vegetale) (13): solamente gli oggetti in plastica che abbiamo prodotto pesano il doppio di tutti gli animali del mondo! La stima, pubblicata su Nature per L'Istituto Israeliano per le Scienze, riporta che i 1.100 miliardi di tonnellate di massa antropica superano i 1.000 dell'attuale biomassa. Sono dati in parte già paventati nel 2018, quando, su PNAS, si scriveva che l'umanità, in termini di peso, rappresenta lo $0,01 \%$ degli esseri viventi, ma, al contempo, incide incredibilmente di più sugli equilibri dell'ecosistema del Pianeta (14). 
È evidente che, se ognuno di noi dovrà fare indubbiamente la sua parte per migliorare le cose di cui sopra, quello che realmente conterà sarà tutti insieme "obbligare" i nostri decisori a prendere delle decisioni non proprio da para ..., come a me sembra che finora abbiano fatto.

Ed ecco, finalmente, che svelo l'arcano dell'incipit di questo breve scritto su come ricorderemo il 2020: se l'uomo del futuro sarà più donna e i cosiddetti decisori saranno formati più da donne che da uomini avremo una speranza in più.

Meditiamo gente, meditiamo, ma davvero!

\section{Bibliografia}

1. Il Colibrì. Veronesi S. 2019. La Nave di Teseo Edizioni, pag. 177.

2. Il Cammino Interiore con Prof. Ernesto Burgio e Prof. Marco Francesconi. Sul canale Youtube degli Stati Generali delle Donne. Online

3. Icenogle T. COVID-19: infection or Autoimmunity. Front Immunol. 2020;11:2055. CrossRef PubMed

4. Rodó X, Curcoll R, Robinson M, et al. Tropospheric winds from northeastern China carry the etiologic agent of Kawasaki disease from its source to Japan. Proc Natl Acad Sci USA. 2014;111(22):7952-7957. CrossRef PubMed

5. Pirozzi CS, Jones BE, VanDerslice JA, Zhang $Y$, Paine R III, Dean NC. Short-Term Air Pollution and Incident Pneumonia. A CaseCrossover Study. Ann Am Thorac Soc. 2018;15(4):449-459. CrossRef PubMed
6. Wilson MR, Lightbody JH, Donaldson K, Sales J, Stone V. Interactions between ultrafine particles and transition metals in vivo and in vitro. Toxicol Appl Pharmacol. 2002;184(3): 172-179. CrossRef PubMed

7. Rosas Pérez I, Serrano J, Alfaro-Moreno E, et al. Relations between PM10 composition and cell toxicity: a multivariate and graphical approach. Chemosphere. 2007;67(6):1218-1228. CrossRef PubMed

8. Buzea C, Pacheco II, Robbie K. Nanomaterials and nanoparticles: sources and toxicity. Biointerphases. 2007;2(4):MR17-MR71. CrossRef PubMed

9. Su W, Wu X, Geng X, Zhao X, Liu Q, Liu T. The short-term effects of air pollutants on influenza-like illness in Jinan, China. BMC Public Health. 2019;19(1):1319. CrossRef PubMed

10. Burgio E. Environment and fetal programming: the origins of some current "pandemics". Journal of Pediatric and Neonatal Individualized Medicine. 2015;4(2):e040237. CrossRef

11. Lombardi M, Bergesio F. GCND per il pianeta. Un forte invito ad agire per tutti: medici, infermieri e pazienti G Clin Nefrol Dial 2021;33:1-2. CrossRef

12. Ragusa A, Svelato A, Santacroce C, et al. Plasticenta: First evidence of microplastics in human placenta. Environ Int. 2021;146:106274. CrossRef PubMed

13. Elhacham E, Ben-Uri L, Grozovski J, Bar-On YM, Milo R. Global human-made mass exceeds all living biomass. Nature. 2020;588(7838):442-444. CrossRef PubMed

14. Bar-On YM, Phillips R, Milo R. The biomass distribution on Earth. Proc Natl Acad Sci USA. 2018;115(25):6506-6511. CrossRef PubMed 